\title{
Solid-liquid exchange between uranium and a synthetic apatite: towards uranium decorporation from bone matrix
}

\author{
Jingxian Wang ${ }^{1,}$, Damien Bourgeois ${ }^{1}$, and Daniel Meyer ${ }^{1}$ \\ ${ }^{1}$ ICSM, LHYS, BP 17171, 30207 Bagnols-sur-Cèze, France
}

\begin{abstract}
Natural uranium exhibits chemical toxicity, especially known with its acute effects on kidney. Simultaneously, it has been proved that uranium accumulates in bones during longterm exposure[1] but its chronical effects on bones are not clear. Particularly the mechanisms associated to accumulation into and release from bones are unknown, which is key to design and test decorporation reagents in future. Bone is a complicated organ, composed of mineralized apatite and organic compounds (mostly type I collagen). Our work is dedicated to the understanding of how uranium is accumulated in the inorganic bone matrix through chemical pathways, and what factors influence the solid/liquid equilibrium between uranium and the bone. To fulfil this goal, apatite materials which mimic bone apatite have been synthesized, with and without uranium. Such apatite materials have been pre-equilibrated with a fluid mimicking blood plasma at physiological $\mathrm{pH}=7.4$.
\end{abstract}

The proposed exchange mechanism of +2 cations $\left(\mathrm{Ca}^{2+}, \mathrm{Zn}^{2+}\right.$, etc. $)$ with bone relies on a two compartment model[2], including ion exchange in the amorphous hydration shell and further ion diffusion into the mineral matrix. From our previous work and other researchers[3-6], bone apatite has high affinity to uranium. However, the two compartment model has not been applied to $U$ exchange, and studies are complicated by the complex speciation of uranium in vivo. U(VI) in physiological fluids forms many inorganic complexes, such as with carbonate and calcium, and organic complexes, instead of "free" $\left\{\mathrm{UO}_{2}{ }^{2+}\right\}[7]$. In our study, U(VI) is introduced in liquid containing carbonate ions in order to investigate the exchanges of $\mathrm{U}(\mathrm{VI})$-carbonato complexes with the synthetic apatite stated above. Both the thermodynamics and the kinetics behaviors have been analyzed, and the exchange reversibility is further investigated. The apatite matrix after interaction with uranium is also characterized with various techniques, including electron microscopy (TEM and SEM) with energy dispersive X-ray spectroscopy, X-ray diffraction and Raman spectroscopy, to locate uranium and specify possible uranium incorporated phases. Furthermore, X-ray adsorption technique has been applied to study the uranium coordination mode on surface and in mineral, especially the uranium-containing complex specification. Finally, a solid/liquid exchange model is set and it will be employed to evaluate the influence of U(VI) chelators in liquid phase on solid/liquid exchange process, towards to further uranium detoxification studies.

${ }^{*}$ Corresponding author: jingxian.wang@,cea.fr 


\section{References}

1. C. Vidaud, D. Bourgeois, D. Meyer, Chem. Res. Toxicol. 25, 1161 (2012)

2. V.E. Badillo-Almaraz, J. Ly, J. Colloid Interface Sci. 258, 27 (2003)

3. D. Bourgeois, B. Burt-Pichat, X. Le Goff, J. Garrevoet, P. Tack, G. Falkenberg, L. Van Hoorebeke, L. Vincze, M.A. Denecke, D. Meyer, C. Vidaud, G. Boivin, Anal. Bioanal. Chem 407, 6619 (2015)

4. G. Chatelain, D. Bourgeois, J. Ravaux, O. Averseng, C. Vidaud, D. Meyer, J. Biol. Inorg. Chem. 20, 497 (2015)

5. P. Thakur, R.C. Moore, G.R. Choppin, Radiochim. Acta 93, 385 (2005)

6. C.C. Fuller, J.R. Bargar, J.A. Davis, Environ. Sci. Technol. 37, 4642 (2003)

7. G. Bernhard, G. Geipel, T. Reich, V. Brendler, S. Amayri, H. Nitsche, Radiochim. Acta 89, 511 (2001) 